\title{
Fecal Microbiota Transplantation for Treating Hepatic Encephalopathy: Experimental and Clinical Evidence and Possible Underlying Mechanisms
}

\author{
Wen-Rui Xie ${ }^{1, \#}$, Xiao-Ya Yang ${ }^{2, \#, ~ H a r r y ~ H u a-X i a n g ~ X i a ~}{ }^{1}$ and Xing-Xiang $\mathrm{He}^{1 *}$ \\ ${ }^{1}$ Department of Gastroenterology, First Affiliated Hospital of Guangdong Pharmaceutical University, Guangzhou, Guangdong Province, \\ China; ${ }^{2}$ Department of Physiology, Guangzhou Health Science College, Guangzhou, Guangdong Province, China; ${ }^{\#}$ Contributed equally
}

\begin{abstract}
Gut microbiota changes play a key role in the pathogenesis of hepatic encephalopathy (HE). Fecal microbiota transplantation (FMT) is an efficient way to manipulate the gut microbiota. This review collects the experimental and clinical evidence that supports the use of FMT in the treatment of HE. Animal experiments showed that the blood ammonia level, mortality and cognitive impairment were decreased when animals with HE were transplanted with the selected gut microbiota or the fecal material from human donor. Successful clinical application of FMT for treating HE was first reported in 2016. A subsequent randomized clinical trial demonstrated further that FMT from a "rationally selected donor" reduced hospitalizations and improved cognition and dysbiosis in patients with recurrent HE. Possible underlying mechanisms of FMT treating HE include restoration of the impaired gut-liver-brain axis, as well as reduction of ammonia production, systemic inflammation and blood-brain barrier permeability.
\end{abstract}

\section{Introduction}

Hepatic encephalopathy (HE), one of the most serious complications of liver dysfunction, is characterized by a broad range of neuropsychiatric symptoms, from subtle fluctuating cognitive impairment to confusion, coma and death. ${ }^{1}$ The pathogenesis of HE has not been completely elucidated. The increase in blood or brain ammonia levels predominates in the overall understanding of HE to date. Ammonia is released from the gut after breakdown of digested proteins by gut bacteria and mucosal enzymes, after which it enters the portal circulation of the liver and is transformed into urea via the urea cycle. When encountering hepatic failure, the accumulated ammonia is shunted into the systemic circulation, which results in hyperammonemia, and consequently leads to the abnormal functions of neurons, and ultimately HE. ${ }^{2}$ The

Keywords: Hepatic encephalopathy; Fecal microbiota transplantation; Gut-liverbrain axis; Ammonia; Blood-brain barrier.

Abbreviations: AE, adverse event; ASF, altered Schaedler flora; BBB, blood-brain barrier; FMT, fecal microbiota transplantation; HE, hepatic encephalopathy; IL, interleukin; MHE, minimal HE; SAE, serious adverse event; SOC, standard of care; TAA, thioacetamide; TLR, Toll-like receptor; TNF, tumor necrosis factor.

Received: July 12, 2018; Revised: October 28, 2018; Accepted: November 12, 2018 *Correspondence to: Xing-Xiang He, Department of Gastroenterology, First Affiliated Hospital of Guangdong Pharmaceutical University, 19 Nonglinxia Road, Guangzhou 510080, Guangdong Province, China. Tel: +86-(0)20-61321457; Fax: +86-(0)20-61326127; E-mail: hexingxiang@gdpu.edu.cn

How to cite this article: Xie W-R, Yang X-Y, Xia HH-X, He X-X. Fecal Microbiota Transplantation for Treating Hepatic Encephalopathy: Experimental and Clinical Evidence and Possible Underlying Mechanisms. J Explor Res Pharmacol 2018;3(4):119124. doi: 10.14218/JERP.2018.00017 accumulated ammonia can pass the blood-brain barrier (BBB), where ammonia and glutamate are then converted into glutamine by astrocytic glutamine synthetase, and the increased glutamine leads to brain edema. ${ }^{2}$ Other factors besides ammonia have been involved in the pathogenesis of HE, including benzodiazepine receptors, ${ }^{3}$ manganese toxicity, ${ }^{4,5}$ neuroinflammatory responses, ${ }^{5}$ oxidative stress,,${ }^{5}$ mercaptans, short fatty acids, lactate, decreased glutaminergic synaptic function and dopamine metabolites. ${ }^{6}$ The use of lactulose, lactulose combined with refaximin, and intravenous L-ornithine-L-aspartate comprises the initial management of $\mathrm{HE}$, which aims to reduce ammonia levels..$^{1,2,7,8}$ Although these treatments may ameliorate HE symptoms in some patients, poor clinical outcome with periods of worsening have been observed in a substantial proportion of patients.

Recently, changes in the gut microbiota (i.e. the decrease of beneficial bacteria and the increase of potentially pathogenic bacteria) are considered to play a key role in the pathogenic mechanism of HE. ${ }^{9}$ Patients with HE may benefit from treatment with some antibiotics, such as rifaximin, or probiotics that act as modulators of gut microbiota. ${ }^{10,11}$ However, long-term application of rifaximin may induce drug resistance and it is possible that probiotics will introduce live bacteria into immunosuppressed patients.

Fecal microbiota transplantation (FMT) is an efficient strategy to manipulate gut microbiota through infusing a healthy donor's stool into the intestine of a sick recipient. In recent years, FMT has shown outstanding clinical efficacy on Clostridium difficile infection, inflammatory bowel disease, irritable bowel syndrome, nonalcoholic fatty liver disease, etc. ${ }^{12-16}$ Moreover, FMT has been explored in the treatment of HE, showing promising efficacy and safety. In this review article, we provide currently available experimental and clini- 
Table 1. Experimental evidence of FMT in treating $\mathrm{HE}$

\begin{tabular}{|c|c|c|}
\hline Study design & Shen et al. ${ }^{7}$ & Wang et al. ${ }^{8}$ \\
\hline \multicolumn{3}{|l|}{ Animals } \\
\hline Recipient & Germ-free mice/C57BL6 mice & Male Sprague-Dawley rats \\
\hline Control & Swiss Webster mice/C57BL6 mice & Male Sprague-Dawley rats \\
\hline Pre-treatment before FMT & $\begin{array}{l}\text { Antibiotics+ intestinal purge } \\
\text { using polyethylene glycol }\end{array}$ & No \\
\hline Feces source & $\begin{array}{l}\text { ASF (from CB17 SCID mice } \\
\text { colonized with ASF) }\end{array}$ & Fecal material from a healthy female donor \\
\hline Route of FMT & Oral gavage & Intestinal intubation \\
\hline \multicolumn{3}{|l|}{ Induction of the HE model } \\
\hline How & TAA injection & $\mathrm{CCl}_{4}$ injection + alcohol feeding \\
\hline When & Before FMT & After FMT \\
\hline \multicolumn{3}{|l|}{ After FMT } \\
\hline Ammonia & $\downarrow$ & $\downarrow$ \\
\hline Mortality & $\downarrow$ & $\downarrow$ \\
\hline Cognitive impairment & $\downarrow$ & $\downarrow$ \\
\hline Hepatic function & / & $\uparrow$ \\
\hline Intestinal mucosal barrier dysfunction & / & $\downarrow$ \\
\hline Systemic proinflammatory response & / & $\downarrow$ \\
\hline Possible mechanisms & / & $\begin{array}{l}\text { Impairing liver inflammation, reducing systemic } \\
\text { inflammation, and improving intestinal mucosal } \\
\text { barrier function. }\end{array}$ \\
\hline
\end{tabular}

Abbreviations: ASF, altered Schaedler flora; $\mathrm{CCl}_{4}$, carbon tetrachloride; FMT: fecal microbial transplant; HE: hepatic encephalopathy; SCID, severe combined immunodeficiency syndrome; TAA, thioacetamide. $\downarrow$, decreased; /, no change.

cal evidence, and then propose the possible underlying mechanisms.

\section{Experimental evidence}

Shen et al. ${ }^{17}$ engineered the gut microbiota to treat hyperammonemia. The researchers selected altered Schaedler flora (ASF), which is low in urease activity, to establish ASF-transplanted mice. The ASF transplantation alleviated the effects of acute and chronic liver injury that had been induced by thioacetamide (TAA). Fecal ammonia levels, mortality and cognitive impairment in response to TAA were significantly reduced in the ASF-transplanted mice, as compared with those in the control mice with conventional microbiota. Another recent study carried out by Wang et al. ${ }^{18}$ also showed positive effects of FMT in HE rats, different from the study by Shen et al. ${ }^{17}$ in that the fecal material used was collected from a healthy female donor (Table 1). ${ }^{7,8}$ In addition to the decline of blood ammonia, mortality and cognitive impairment, FMT decreased liver inflammation and damage, and attenuated intestinal permeability (Table 1). ${ }^{18}$ The findings from these humanized versions of FMT in animal models give hope that FMT can clinically reduce hyperammonemia in patients with $\mathrm{HE}$.

\section{Clinical evidence}

Both culture-based and culture-independent studies of microbiota have demonstrated that gut microbiota is altered in cirrhosis and
HE. ${ }^{9,19,20}$ Clinical studies have also further confirmed the associations between changes in gut microbiota and HE. ${ }^{21,22}$ The studies by Bajaj et al. ${ }^{21,22}$ showed that the model for end-stage liver disease score, a scoring system to measure the severity of cirrhosis, was positively correlated with potentially pathogenic taxa, such as Enterobacteriaceae, Enterococceae and Staphylococcae, and negatively correlated with Clostridiales XIV, Ruminococcaceae and Lachnospiraceae in gut microbiota. These findings indicate that when beneficial bacteria decrease and potentially pathogenic bacteria increase in cirrhosis, the gut microbiota balance will be broken, which then plays pathogenic roles in HE progression.

Given the close association between microbiota dysbiosis and HE, Canadian doctors, Kao and her colleagues, ${ }^{23}$ first applied FMT in an overt HE patient and reported their results in 2016, which indicated significant cognitive improvement. The 57-year-old man with HE was given four weekly FMTs, at weeks 1-2, 3, 4 and 7 , collected from a "universal" stool donor, whereas the patient's diet and lactulose dose remained the same throughout the study. The patient's inhibitory control rest and the Stroop test scores returned to normal by week 4, but reverted to the level before FMT at week 14, implying that repeated therapy is required to preserve the beneficial effect of FMT. No FMT-related adverse events (AEs) or infectious complications occurred. ${ }^{23}$

In 2017, Bajaj and colleagues published an open-label randomized clinical trial of single FMT versus the standard of care (SOC) in patients with recurrent HE. ${ }^{24}$ In this FMT trial, the stool donor was a so-called "rationally derived stool donor", who had been selected based on cross-sectional HE microbiome data. A 5-day broad-spectrum antibiotic pretreatment was given to the pa- 
Table 2. Efficacy and safety of an open-label randomized clinical trial of single FMT versus the SOC in patients with recurrent $\mathrm{HE}^{14}$

\begin{tabular}{|c|c|c|}
\hline & SOC $\operatorname{arm}(n=10)$ & FMT $\operatorname{arm}(n=10)$ \\
\hline $\begin{array}{l}\text { Proportion of patients with FMT- } \\
\text { related SAEs at day } 150\end{array}$ & $80 \%$ & $20 \%$ \\
\hline Death & 0 & 0 \\
\hline Hospitalizations for recurrent $\mathrm{HE}$ & $60 \%$ & 0 \\
\hline ER visits/transmissible infection & 0 & 0 \\
\hline $\begin{array}{l}\text { Cognition improvement (PHES and } \\
\text { EncephalApp-Stroop Test) }\end{array}$ & Yes & No \\
\hline \multicolumn{3}{|l|}{ Changes in cirrhosis severity } \\
\hline MELD scores & No changes & $\begin{array}{l}\text { Transiently worsened post-antibiotics; } \\
\text { Reverted to baseline post-FMT }\end{array}$ \\
\hline Albumin & No changes & No changes \\
\hline $\begin{array}{l}\text { Changes in liver function (AST, ALT, albumin or } \\
\text { hemoglobin) and WBC count }\end{array}$ & No changes & No changes \\
\hline \multicolumn{3}{|l|}{ Changes in microbiota composition and function } \\
\hline Diversity & No changes & $\begin{array}{l}\text { Post-antibiotics-reduced significantly } \\
\text { Post-FMT-increased and returned } \\
\text { to the pre-FMT values }\end{array}$ \\
\hline Beneficial taxa & No changes & $\begin{array}{l}\text { A relative increase in Lactobacillaceae } \\
\text { and Bifidobacteriaceae; } \\
\text { The relative abundance of Lachnospiraceae } \\
\text { and Ruminococcaceae reduced } \\
\text { post-antibiotics and recovered } \\
\text { post-FMT in all but } 2 \text { patients. }\end{array}$ \\
\hline Solicited AE events & $\begin{array}{l}\text { Linked to liver-related complications: } \\
\text { Mental status alteration }(n=6) \\
\text { Pneumonia }(n=1) \\
\text { Chest pain }(n=1) \\
\text { Portal vein thrombus }(n=1) \\
\text { Anemia }(n=1) \\
\text { Gastroenteritis }(n=1) \\
\text { Variceal bleeding }(n=2)\end{array}$ & $\begin{array}{l}\text { Unrelated to FMT: } \\
\text { Acute kidney injury }(n=1) \text { (responded } \\
\text { within } 24 \text { h to intravenous hydration) } \\
\text { Chest pain }(n=1) \text { (ruled out for } \\
\text { an acute cardiac event) }\end{array}$ \\
\hline
\end{tabular}

Data are derived from Bajaj et al.14 "Fecal microbiota transplant from a rational stool donor improves hepatic encephalopathy: A randomized clinical trial." Hepatology 2017; 66:1727-1738. Abbreviations: AE, adverse event; ALT, alanine aminotransferase; AST, aspartate aminotransferase; EncephalApp - Stroop test, a convenient method to test for cognitive dysfunction; ER, emergency room; FMT: fecal microbial transplantation; HE: hepatic encephalopathy; MELD, model for end-stage liver disease; PHES, psychometric hepatic encephalopathy score; SAE, serious adverse event; SOC: standard of care; WBC, white blood cell.

tients randomized to receive a single FMT enema (the FMT arm), whereas no antibiotic pretreatment was given to those randomized to receive SOC (the SOC arm). Patients in both arms were on steady doses of lactulose and rifaximin during the study. The primary outcome of this trial was safety of FMT, as determined by FMT-related serious adverse events (SAEs) at day 150, which was defined by a composite endpoint of death, hospitalizations, emergency room visits or transmissible infections, as defined by the Federal Drug Administration. Changes in cognitive functions, cirrhosis severity, microbiota composition and function, and solicited AEs were also evaluated. From the clinical findings (summarized in Table 2), Bajaj et al. ${ }^{14,24}$ concluded that FMT from a rationally selected donor reduced hospitalizations, and improved cognition and dysbiosis in patients with recurrent HE, indicating that FMT is safe.

Although there are some weak points in the sample size, length of follow-up, donor selection, and lack of long-term safety data, ${ }^{25-}$ 27 this trial proved FMT as a feasible therapeutical treatment approach for HE. However, more data on the efficacy, durability and long-term safety are required to support FMT as the front-line treatment option for HE. ${ }^{25}$

Possible underlying mechanisms

The pathogenesis of HE is complicated and remains incompletely understood. However, the impaired gut-liver-brain axis, hyperammonemia, systemic inflammation and the increasing BBB permeability are believed to contribute to the pathogenesis (Fig. 1). Based on the current limited published data, we propose here the possible underlying mechanisms of FMT for its therapeutic role in HE (Fig. 1).

\section{Restoration of the impaired gut-liver-brain axis}

A small-scale study by Bajaj et al. ${ }^{22}$ showed that the Alcaligeneceae and Porphyromonadaceae dominant gut microbiome was positively linked with cognitive impairment. In a recent large-scale 


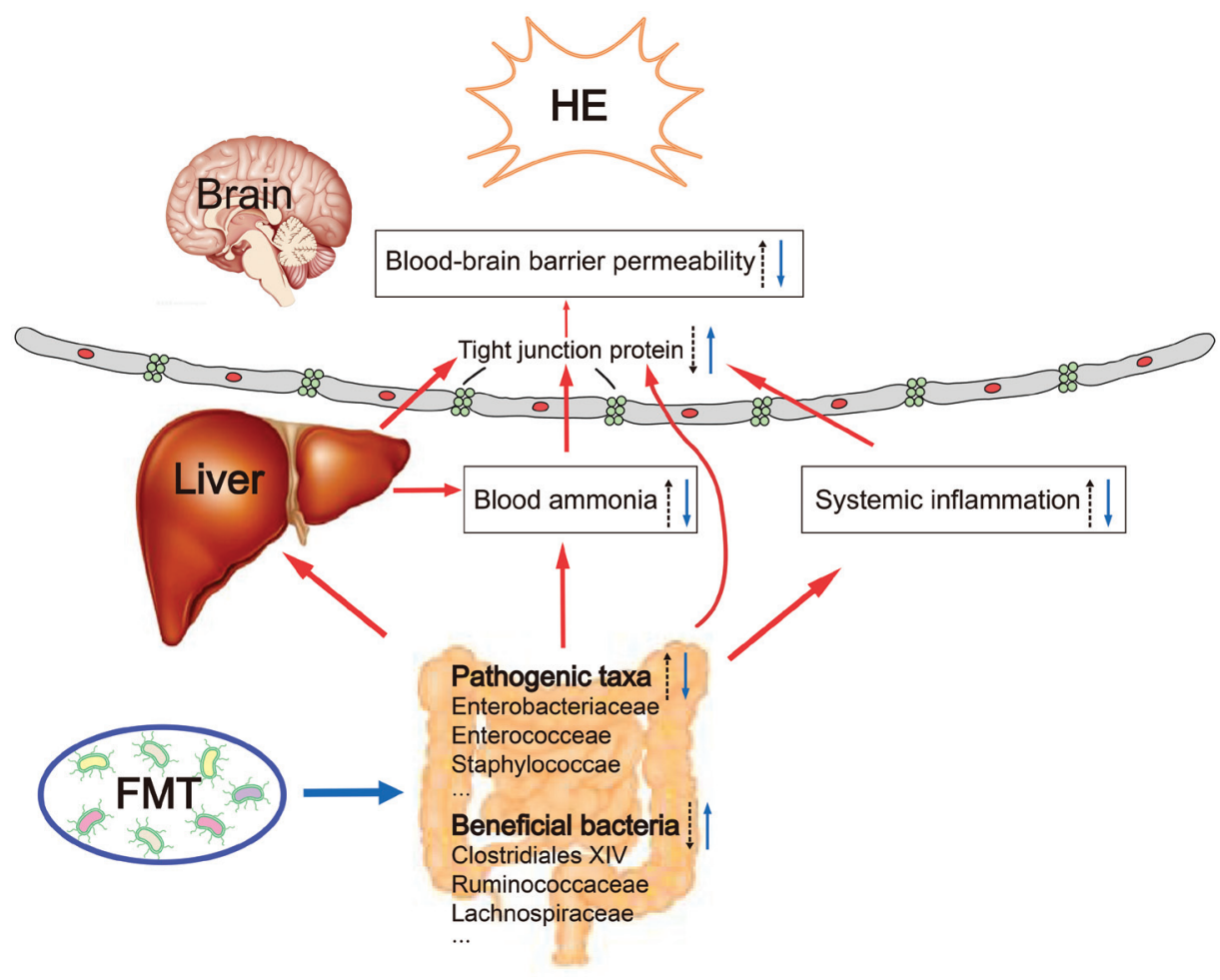

Fig. 1. Possible underlying mechanisms of FMT for treating HE. The gut microbiota imbalance resulted from reduction of beneficial bacteria and increase of potentially pathogenic bacteria, which leads to gut-liver-brain axis impairment, hyperammonemia, systemic inflammation and increasing blood-brain barrier permeability, ultimately contributing to the development of HE. FMT, an efficient way to manipulate gut microbiota, treats HE possibly via restoration of the impaired gut-liver-brain axis, and reduction of ammonia production, systemic inflammation and blood-brain barrier permeability. Abbreviations: FMT, fecal microbial transplantation; HE, hepatic encephalopathy. $\uparrow$ increase in patients with HE; $\downarrow$ decrease in patients with HE; $\downarrow$ decrease after FMT; $\uparrow$ increase after FMT.

study, Ahluwalia et al. ${ }^{28}$ explored the linkage between gut microbiome and astrocytic and neuronal changes in brain function in patients with cirrhosis. Autochthonous taxa were found to negatively correlate with magnetic resonance spectroscopy and hyperammonemia-associated astrocytic changes, while Enterobacteriaceae showed a positive correlation with those changes. Porphyromonadaceae showed the correlation with neuronal changes on diffusion tensor imaging which demonstrates impaired axonal integrity and edema, but no linkages with ammonia. These findings indicate that the impaired gut-liver-brain axis in cirrhosis plays a crucial role in the ultimate pathogenesis of HE. Accordingly, FMT, a promising therapy to restore a healthy microbiota, may reduce HE recurrence via restoring the impaired gut-liver-brain axis.

\section{Ammonia reduction}

Hyperammonemia is a classical factor in the pathogenesis of HE. Human gut bacteria produce urease, which hydrolyzes urea into carbon dioxide and ammonia. Accumulated ammonia, which occurs in patients with liver injury, chronic liver disease or urea cycle defects, is associated with damage to the central nervous system in patients, resulting in HE. ${ }^{29}$ The above-mentioned murine experiment by Shen et al. ${ }^{17}$ showed a long-term reduction in fecal urease activity and ammonia production upon transplanting ASF into mice previously colonized with other organisms. A study of 26 minimal HE (MHE) patients revealed that Streptococcus salivarius, the gut urease-containing bacterium, was present in cirrhotic patients with and without MHE, but was absent in the normal group. The abundance of Streptococcus salivarius was positively linked with ammonia accumulation in cirrhotic patients with MHE. ${ }^{30}$ Therefore, modulation of the gut bacteria related to urease activity, in order to reduce ammonia production, may act as a possible mechanism of FMT for treating HE.

\section{Decrease in systemic inflammation}

Mounting evidence indicates that systemic inflammation leads to exacerbation of HE. ${ }^{11,31-33}$ Bajaj et al. ${ }^{22}$ found key linkages between the altered flora and inflammation, endotoxemia and poor cognition in patients with HE. In the above-mentioned study by Wang et al., ${ }^{18}$ on FMT treatment using HE rats, liver expression of Toll-like receptor (TLR) 4 and TLR9 was reduced after FMT, accompanied by reduction in levels of proinflammatory factors, e.g. interleukin (IL)-1 $\beta$, IL- 6 and tumor necrosis factor (TNF)- $\alpha$, indicating that FMT attenuates systemic inflammation. Therefore, alleviating systemic inflammation emerges as a possible mechanism of FMT treating HE.

\section{Reduction of $B B B$ permeability}

An intact and tightly regulated BBB performs as a "guard" to protect against the passage of harmful molecules in blood into the brain parenchyma. The BBB is composed of capillary endothe- 
lial cells, which are sealed by astrocytes, pericytes, and tight junctions. Tight junction proteins restrain the diffusion of water-soluble substances from blood to the brain in the paracellular pathway. ${ }^{34,35}$ When tight junctions are disrupted by disease or drugs, BBB function and even the central nervous system will be impaired. ${ }^{35,36}$ In the bile duct ligation model of HE, BBB permeability was found to be exacerbated and the expression of tight junction proteins was down-regulated, suggesting their roles in the pathogenesis of HE. ${ }^{37}$ Braniste et al. ${ }^{36}$ compared germ-free mice with pathogen-free mice with a normal gut flora and reported that lack of gut microbiota was related to an increase in BBB permeability and a change in tight junction protein expression, and $\mathrm{BBB}$ permeability was decreased in germ-free mice after transplantation of pathogen-free gut flora. Therefore, up-regulating tight junction proteins and increasing $\mathrm{BBB}$ permeability is a possible mechanism of FMT treating HE.

\section{Future research directions}

As gut microbiota changes play a pivotal role in the pathogenesis of HE. It is necessary to confirm the precise bacteria that are involved in pathogenesis of HE, which could guide the bacterial profile included in FMT. The efficacy, durability and long-term safety of FMT need to be assessed further in the future, using a well-designed randomized clinical trial with a larger sample size and longer follow-up period. Besides, the underlying mechanisms of FMT for its therapeutic role in HE also need to be elucidated. Based on the mechanisms of HE, we hypothesize that the possible underlying mechanisms of FMT in treating HE may include restoration of the impaired gut-liver-brain axis, and reduction of ammonia production, systemic inflammation and BBB permeability.

\section{Conclusions}

Gut microbiota changes play a key role in the pathogenesis of HE. Recent experimental and clinical evidence suggests FMT as a feasible, efficacious and safe therapeutical treatment approach for HE. However, before FMT can be considered as the front-line treatment option for HE, more extensive experimental and clinical studies are required to determine the precise bacteria to be included in FMT, the efficacy, durability and long-term safety of FMT, and to elucidate the underlying mechanisms. When the efficacy and safety of FMT in treating HE are confirmed, more and more patients with $\mathrm{HE}$ will benefit from the treatment.

\section{Acknowledgments}

This work was supported by Traditional Chinese Medicine Bureau of Guangdong Province (20151283) and Department of Education of Guangdong Province (2014KQNCX113).

\section{Conflict of interest}

The authors have no conflict of interests related to this publication.

\section{Author contributions}

Manuscript writing (WRX, XYY, HXX, XXH).

\section{References}

[1] Swaminathan M, Ellul MA, Cross TJ. Hepatic encephalopathy: current challenges and future prospects. Hepat Med 2018;10:1-11. doi:10.2147/HMER.S118964.

[2] Wijdicks EF. Hepatic encephalopathy. N Engl J Med 2016;375:16601670. doi:10.1056/NEJMra1600561.

[3] Butterworth RF, Giguere JF, Michaud J, Lavoie J, Layrargues GP. Ammonia: key factor in the pathogenesis of hepatic encephalopathy. Neurochem Pathol 1987;6:1-12. doi:10.1007/bf02833598.

[4] Rivera-Mancia S, Rios C, Montes S. Manganese accumulation in the CNS and associated pathologies. Biometals 2011;24:811-825. doi:10.1007/s10534-011-9454-1.

[5] McPhail MJ, Bajaj JS, Thomas HC, Taylor-Robinson SD. Pathogenesis and diagnosis of hepatic encephalopathy. Expert Rev Gastroenterol Hepatol 2010;4:365-378. doi:10.1586/egh.10.32.

[6] Butterworth RF. Neurosteroids in hepatic encephalopathy: novel insights and new therapeutic opportunities. J Steroid Biochem Mol Biol 2016;160:94-97. doi:10.1016/j.jsbmb.2015.11.006.

[7] Acharya C, Bajaj JS. Current management of hepatic encephalopathy. Am J Gastroenterol 2018; doi:10.1038/s41395-018-0179-4.

[8] Flamm SL. Complications of cirrhosis in primary care: recognition and management of hepatic encephalopathy. Am J Med Sci 2018;356:296-303. doi:10.1016/j.amjms.2018.06.008.

[9] Bajaj JS. The role of microbiota in hepatic encephalopathy. Gut Microbes 2014;5:397-403. doi:10.4161/gmic.28684.

[10] Ianiro G, Tilg H, Gasbarrini A. Antibiotics as deep modulators of gut microbiota: between good and evil. Gut 2016;65(11):1906-1915. doi:10.1136/gutjnl-2016-312297.

[11] Dhiman RK. Gut microbiota and hepatic encephalopathy. Metab Brain Dis 2013;28:321-326. doi:10.1007/s11011-013-9388-0.

[12] Borody T, Fischer M, Mitchell S, Campbell J. Fecal microbiota transplantation in gastrointestinal disease: 2015 update and the road ahead. Expert Rev Gastroenterol Hepatol 2015;9:1379-1391. doi:10. 1586/17474124.2015.1086267.

[13] Costello SP, Soo W, Bryant RV, Jairath V, Hart AL, Andrews JM. Systematic review with meta-analysis: faecal microbiota transplantation for the induction of remission for active ulcerative colitis. Aliment Pharmacol Ther 2017;46:213-224. doi:10.1111/apt.14173.

[14] Quraishi MN, Widlak M, Bhala N, Moore D, Price M, Sharma N, et al. Systematic review with meta-analysis: the efficacy of faecal microbiota transplantation for the treatment of recurrent and refractory Clostridium difficile infection. Aliment Pharmacol Ther 2017;46:479493. doi:10.1111/apt.14201.

[15] Laffin M, Millan B, Madsen KL. Fecal microbial transplantation as a therapeutic option in patients colonized with antibiotic resistant organisms. Gut Microbes 2017;8:221-224. doi:10.1080/19490976.201 6.1278105

[16] Millan B, Laffin M, Madsen K. Fecal microbiota transplantation: beyond clostridium difficile. Curr Infect Dis Rep 2017;19:31. doi:10.1007/s11908-017-0586-5.

[17] Shen TC, Albenberg L, Bittinger K, Chehoud C, Chen YY, Judge CA, et al. Engineering the gut microbiota to treat hyperammonemia. J Clin Invest 2015;125:2841-2850. doi:10.1172/JCI79214.

[18] Wang WW, Zhang Y, Huang XB, You N, Zheng L, Li J. Fecal microbiota transplantation prevents hepatic encephalopathy in rats with carbon tetrachloride-induced acute hepatic dysfunction. World J Gastroenterol 2017;23:6983-6994. doi:10.3748/wjg.v23.i38.6983.

[19] Liu Q, Duan ZP, Ha DK, Bengmark S, Kurtovic J, Riordan SM. Synbiotic modulation of gut flora: effect on minimal hepatic encephalopathy in patients with cirrhosis. Hepatology 2004;39:1441-1449. doi:10.1002/hep.20194.

[20] Riggio O, Varriale M, Testore GP, Di Rosa R, Di Rosa E, Merli M, et al. Effect of lactitol and lactulose administration on the fecal flora in cirrhotic patients. J Clin Gastroenterol 1990;12:433-436. doi:10.1097/00004836-199008000-00016.

[21] Bajaj JS, Heuman DM, Hylemon PB, Sanyal AJ, White MB, Monteith $\mathrm{P}$, et al. Altered profile of human gut microbiome is associated with cirrhosis and its complications. J Hepatol 2014;60:940-947. doi:10.1016/j.jhep.2013.12.019. 
J Explor Res Pharmacol

[22] Bajaj JS, Ridlon JM, Hylemon PB, Thacker LR, Heuman DM, Smith S, et al. Linkage of gut microbiome with cognition in hepatic encephalopathy. Am J Physiol Gastrointest Liver Physiol 2012;302:G168-G175. doi:10.1152/ajpgi.00190.2011.

[23] Kao D, Roach B, Park H, Hotte N, Madsen K, Bain V, et al. Fecal microbiota transplantation in the management of hepatic encephalopathy. Hepatology 2016;63:339-340. doi:10.1002/hep.28121.

[24] Bajaj JS, Kassam Z, Fagan A, Gavis EA, Liu E, Cox IJ, et al. Fecal microbiota transplant from a rational stool donor improves hepatic encephalopathy: a randomized clinical trial. Hepatology 2017;66(6):17271738. doi:10.1002/hep.29306.

[25] Tandon P, Madsen K, Kao D. Fecal microbiota transplantation for hepatic encephalopathy: Ready for prime time? Hepatology 2017;66:1713-1715. doi:10.1002/hep.29396.

[26] Mullish BH, McDonald JAK, Thursz MR, Marchesi JR. Fecal microbiota transplant from a rational stool donor improves hepatic encephalopathy: A randomized clinical trial. Hepatology 2017;66:1354-1355. doi:10.1002/hep.29369.

[27] Karakan T. Fecal microbiota transplantation for treating recurrent hepatic encephalopathy: Ready for clinical application? Turk J Gastroenterol 2017;28:425-426. doi:10.5152/tjg.2017.18817.

[28] Ahluwalia V, Betrapally NS, Hylemon PB, White MB, Gillevet PM, Unser $A B$, et al. Impaired gut-liver-brain axis in patients with cirrhosis. Sci Rep 2016;6:26800. doi:10.1038/srep26800.

[29] Rai R, Saraswat VA, Dhiman RK. Gut microbiota: its role in hepatic encephalopathy. J Clin Exp Hepatol 2015;5:S29-36. doi:10.1016/j. jceh.2014.12.003.

[30] Zhang Z, Zhai H, Geng J, Yu R, Ren H, Fan H, et al. Large-scale survey
Xie WR. et al: FMT for treating hepatic encephalopathy

of gut microbiota associated with MHE via $16 \mathrm{~S}$ rRNA-based pyrosequencing. Am J Gastroenterol 2013;108:1601-1611. doi:10.1038/ ajg.2013.221.

[31] Shawcross DL, Sharifi Y, Canavan JB, Yeoman AD, Abeles RD, Taylor NJ, et al. Infection and systemic inflammation, not ammonia, are associated with Grade 3/4 hepatic encephalopathy, but not mortality in cirrhosis. J Hepatol 2011;54:640-649. doi:10.1016/j. jhep.2010.07.045.

[32] Shawcross DL, Wright G, Olde Damink SWM, Jalan R. Role of ammonia and inflammation in minimal hepatic encephalopathy. Metab Brain Dis 2007;22:125-138. doi:10.1007/s11011-006-9042-1.

[33] Wright G, Jalan R. Ammonia and inflammation in the pathogenesis of hepatic encephalopathy: Pandora's box? Hepatology 2007;46:291294. doi:10.1002/hep.21843.

[34] Abbott NJ, Patabendige A, Dolman DEM, Yusof SR, Begley DJ. Structure and function of the blood-brain barrier. Neurobiol Dis 2010;37:13-25. doi:10.1016/j.nbd.2009.07.030.

[35] Hawkins BT, Davis TP. The blood-brain barrier/neurovascular unit in health and disease. Pharmacol Rev 2005;57:173-185. doi:10.1124/ pr.57.2.4.

[36] Braniste V, Al-Asmakh M, Kowal C, Anuar F, Abbaspour A, Toth M, et al. The gut microbiota influences blood-brain barrier permeability in mice. Sci Transl Med 2014;6:263ra158. doi:10.1126/scitransImed.3009759.

[37] Dhanda S, Sandhir R. Blood-brain barrier permeability is exacerbated in experimental model of hepatic encephalopathy via MMP-9 activation and downregulation of tight junction proteins. Mol Neurobiol 2018;55(5):3642-3659. doi:10.1007/s12035-017-0521-7. 